\title{
Vitamin D intakes and blood pressure in Caucasian and South Asian women aged 20-64 years - baseline analysis of the D2-D3 study
}

\author{
L. Wilson, K. Hart, S. Lanham-New and L. Tripkovic \\ Department of Nutritional Sciences, Faculty of Health and Medical Sciences, University of Surrey, Guildford GU2 7XH, UK
}

High blood pressure affects $40 \%$ of adults worldwide and is one (preventable) contributing factor in the complex aetiology of cardiovascular disease. In cross-sectional studies, an inverse association between vitamin D status [25(OH)D levels] and blood pressure has been shown $^{(1,2)}$, and recent research has indicated that supplementation with vitamin D can lead to a significant reduction in systolic blood pressure, in a dose dependant manner ${ }^{(3)}$. Our recent findings ${ }^{(4)}$ investigating associations between dietary intakes of vitamin D and blood pressure measures showed no associations, but were based on a small population number $(n 88)$. Therefore, the aim of this study was to investigate and compare the association between dietary vitamin D intake and blood pressure in a larger population of women from both Caucasian $(\mathrm{Cn})$ and South Asian (SA) communities, taking into consideration anthropometrics and other dietary influences.

A total of 335 women ( $n 245 \mathrm{Cn}, n 90 \mathrm{SA}$ ) aged 20-64 years were recruited from the Surrey area as part of the D2-D3 study (Vitamin D Food Fortification Trial comparing Vitamin D2 vs. Vitamin D3). Participants had anthropometric and blood pressure measurements conducted, and also completed a four-day food diary, which was analysed using DietPlan6. Blood pressure measurements were taken in rested participants and the mean of three measurements were taken to determine systolic blood pressure (SBP), diastolic blood pressure (DBP), mean arterial pressure (MAP), pulse pressure (PP) and heart rate (HR). Analyses were carried out as whole group and between ethnic/age groups (20-34, 35-49 and 50-64 yrs) and body mass index (BMI) categories $\left(18-24.9 \mathrm{~kg} / \mathrm{m}^{2} \mathrm{and} 25-30 \mathrm{~kg} / \mathrm{m}^{2}\right)$.

The mean age and BMI of the study population (n335) were $43.6 \pm 12.3$ years and $24.1 \pm 3.8 \mathrm{~kg} / \mathrm{m}^{2}$ respectively, and the mean SBP, DBP, MAP, PP and HR were $118 \pm 15 \mathrm{mmHg}, 78 \pm 10 \mathrm{mmHg}, 92 \pm 11 \mathrm{mmHg}, 40 \pm 9 \mathrm{mmHg}$ and $68 \pm 10 \mathrm{bpm}$ respectively.

When comparing anthropometrics and blood pressure measurements between ethnic groups, the SA women had a significantly lower age (SA:37.3 \pm 10.4 yrs vs. Cn:46.0 \pm 12.1 yrs, $P<0.001$ ), but higher BMI (SA:25.3 \pm 4.4 vs. Cn:23.6 $\pm 3.5, P<0.001)$, body fat percentage (SA: $32.6 \pm 7.0$ vs. $\mathrm{Cn}: 29.0 \pm 6.3, p=0.000)$ and waist-hip ratio (SA:0.84 $\pm 0.08 v s . \mathrm{Cn}: 0.79 \pm 0.07, P<0.001)$ than the Cn women. Despite these differences in anthropometrics, the SA women had lower SBP (SA:113 \pm 13 vs. Cn:120 $\pm 15 P<0.001$ ), PP $(\mathrm{SA}: 36.5 \pm 7.4 v s$. Cn: $41.4 \pm 8.8 p=0.000)$ and MAP (SA:88.6 \pm 10.5 vs. Cn:92.5 $\pm 10.8 P<0.003)$ than Cn women, but higher HR (SA: $70.7 \pm 9.0$ vs. $\mathrm{Cn}: 67.6 \pm 9.6 P<0.008)$.

Dietary intakes did vary between the ethnic groups, although macro-nutrient intakes were not significantly different (data not presented). Vitamin D intakes were also not significantly different between the groups (SA:2.2 $\pm 2.1 \mu \mathrm{g} / \mathrm{d} v \mathrm{~s}$. Cn:2.7 $\pm 2.3 \mu \mathrm{g} / \mathrm{d})$. These dietary vitamin $\mathrm{D}$ intakes showed no correlation to any measures of blood pressure when analysing the different groups or sub-group analysis taking into account ethnicity/BMI/age.

This work highlights the low dietary vitamin D intakes in both Cn and SA women in the UK. At these low levels, dietary vitamin D intakes show no correlation to blood pressure measures. Our results, alongside RCTs showing an effect of supplementary vitamin D on blood pressure, suggest that intakes of vitamin D at levels achievable without the use of supplementation, are not sufficient to influence blood pressure. Further analysis is underway in the D2-D3 study cohort examining vitamin D status and blood pressure (baseline/change).

The D2-D3 Study is funded by the BBSRC DRINC Programme; LW is recipient of a BBSRC PhD Scholarship.

1. Burgaz et al (2011) J of Hypertension, 29, 636-645.

2. Pittas et al. (2010) Ann Intern Med 152, 307-314.

3. Forman et al. (2013) Hypertension 61, 779-785.

4. Wilson et al. (2013) Proceedings of the Nutrition Society 2013 (In the Press). 